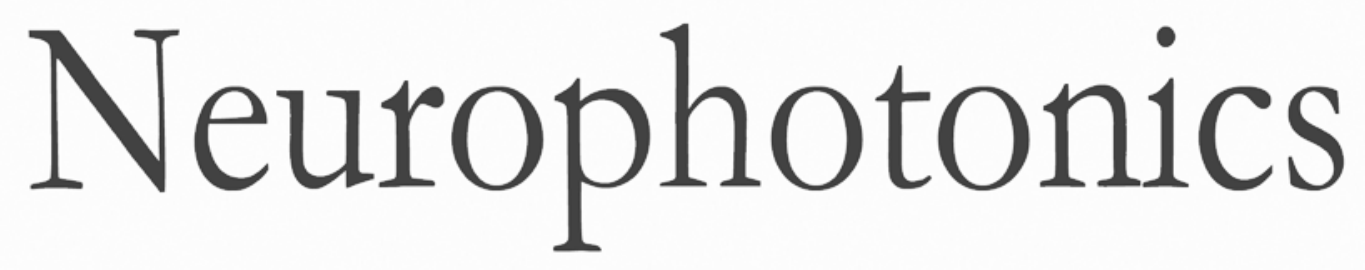

Neurophotonics.SPIEDigitalLibrary.org

\title{
Looking back at fNIRS 2018
}

Lisa Kobayashi Frisk

Gentaro Taga

Turgut Durduran 


\title{
Looking back at fNIRS 2018
}

\author{
Lisa Kobayashi Frisk \\ The Institute of Photonic Sciences (ICFO) \\ Barcelona, Spain \\ E-mail: Lisa.Kobayashi@icfo.eu \\ Gentaro Taga \\ The University of Tokyo \\ Tokyo, Japan \\ Turgut Durduran \\ The Institute of Photonic Sciences (ICFO) \\ Barcelona, Spain
}

From the $5^{\text {th }}$ to $8^{\text {th }}$ of October 2018 , Gentaro Taga, as the local organizer and conference co-chair, and Turgut Durduran, as the program chair, welcomed nearly 450 researchers in functional near infrared spectroscopy (fNIRS) from 29 countries around the world to the biennial conference of the Society for fNIRS at the University of Tokyo. A variety of topics within the fNIRS field of research were presented throughout the four days. These topics included a range of experimental studies in the clinical and life sciences and technological developments, including novel fast diffuse correlation spectroscopy (DCS) systems and improvements in wearable fNIRS devices. In addition, three finalists (all women: Kaja Jasinska, Lauren Emberson, and Sabrina Brigadoi) for the Early Investigator Award presented their work during the conference. The winner, Kaja Jasinska, was awarded at a Japanese taiko drum performance for her impressive and ambitious research.

In the keynote speech, Martin Wolf provided an overview of the achievements, challenges, and possible solutions for the assessment of brain activity by fNIRS and the measurement of absolute values of cerebral tissue oxygenation. While NIRS has been used in clinical research for the past two decades, there is still controversy over the origins and extracerebral contamination in the optical signals. The importance of these complications was highlighted in this year's fNIRS conference. The proper analysis of acquired fNIRS data to correct for motion and physiological artefacts was a major focus of the morning tutorial sessions (along with other crucial issues) where users had the chance to learn from developers.

Different sessions covered various topics of analysis, including algorithms for removing motion artefacts, and using extracerebral signals as a guide for removing systemic signals from fNIRS data. Systemic low frequency oscillations (sLFO), a signal with a frequency of approximately $0.1 \mathrm{~Hz}$, were another popular topic deserving of attention. Yunjie Tong presented his group's work (Yao et al.) on using fMRI and fNIRS resting state data to verify the extracerebral origin of these signals. He also reported findings on the influence these sLFOs exert on the fNIRS signals measured at specific optode positions corresponding to the highly vascularized tissue in the brain. A system designed with the intention of detecting sLFOs was presented ( $\mathrm{Li}$ et al.). In the same vein,

(c) The Authors. Published by SPIE under a Creative Commons Attribution 4.0 Unported License. Distribution or reproduction of this work in whole or in part requires full attribution of the original publication, including its DOI. another work investigated the effect of vascular structures on the fNIRS signal (Benitez et al.). The results indicated an urgent need to understand how the entire physiology affects the hemodynamic signals that are measured by fNIRS.

A growing number of researchers are utilizing the basic advantages of NIRS technology (affordable, portable, noninvasive) to study the physical effects of poverty and adversity on brain development in developing countries (the Global fNIRS initiative). The effects of malnutrition on brain development are being studied separately by University College London and Harvard Medical School in The Gambia and Guinea-Bissau, respectively. In the Brain Imaging for Global Health Project, Gambian infants are being studied from birth to two years of age to understand the impact of malnutrition on very early brain development. The Harvard group is using NIRS and DCS to determine the effects of a nutritional supplement on the neurodevelopmental growth in children. In Côte d'Ivoire, severe adversity which hinders many children from receiving proper education is a widespread problem. In this study, fNIRS is being used to understand how this adversity in childhood affects brain development that might hinder literacy (Jasinska et al.). A separate study used fNIRS to study the effect of early adversity on brain health in rural India (Wijeakumar et al.).

As in previous years, fNIRS continues to be a popular and useful technique for studying the development of various functional networks during early development. Diffuse optics is particularly well-suited to child development studies, as a variety of protocols that might be inconvenient to perform in more common neuroimaging techniques, such as MRI, are more easily implemented with fNIRS. Fumitaka Homae overviewed progress on functional connectivity studies using fNIRS to elucidate the functional development of the brain. Hajing Niu also gave an overview of application of network theory to fNIRS functional connectivity analysis. Topics presented in this year's conference included the mapping of functional networks during sleep (Bulgarelli et al.), while listening to unfamiliar sounds (Hoshino et al.), while consolidating memory (Benavides-Varela et al.), during joint-attention tasks (Gerloff et al.), and under motor stimulus (Pagliazzi et al.). These neonatal and pediatric developmental fNIRS studies give insight into how the brain matures during the first years, months, and even days of life. Better understanding of this maturation is not only scientifically interesting, but can also hold clinical significance for early detection of various conditions such 
as cerebral palsy and autism. To this end, Zhao et al. have developed a high-density diffuse optical tomography system specifically designed for imaging the brains of newborns. While mapping of the fNIRS signal provides information on the locations of functional activation, Scholkmann et al. characterized the hemodynamic response to visual stimulation of preterm neonates. Their results show that the shape of the hemodynamic response, not only the location, is also an indicator of the development of the neonate.

Diffuse correlation spectroscopy proved to be an area gaining traction, with a marked increase in the amount of DCS work presented, beginning with an inspiring invited talk by one of the pioneers of the method, Arjun Yodh. DCS research presented throughout the conference included both technical innovations and clinical applications. Novel technical results included the presentation of a new hardware correlator capable of acquiring correlation curves at up to $100 \mathrm{~Hz}$ allowing for measurement of pulsatile blood flow, and with it the possibility for exploring new biomarkers in various pathologies (Fischer et al.). Time-domain DCS systems capable of depth sensitive measurements were also presented. These systems use pulsed lasers to time gate the DCS signals. By adjusting the time gate that is used, onesource-detector separation can be used to achieve depth resolved signals. Results from depth sensitive time domain DCS systems were presented (Pagliazzi et al., Samaei et al.), as well as results from simulations aimed at improving the technique to achieve the highest contrast between cerebral blood flow in the deep cortical layers of the human head (Qiu et al.). Blackwell et al. are developing a fully wearable time domain DCS helmet with an end goal of incorporating 100 different optode positions. DCS systems typically utilize lasers with wavelengths around 785. However, infrared light at $1064 \mathrm{~nm}$ provides improved penetration due to a reduced overall extinction. Additionally, using infrared light allows higher injection power, and the lower scattering coefficient in the longer wavelengths means a longer autocorrelation decay time leading to better SNR and a better fit of CBF. Therefore, theoretically, using longer wavelengths in DCS should allow for improvements in the signal quality of DCS measurements. However, in order to efficiently implement such a system for a clinical measurement requires the development of cheap and user-friendly detectors (Wu et al.).

In addition to technical developments in DCS, several experimental studies using the technique were presented.
The presented topics ranged from animal studies, studies on the human brain with different pathologies, to a study on skeletal muscle blood flow. In animals, DCS was validated on piglets using PET and a new conversion mechanism for ease of comparison between the two techniques was developed (Giovannella et al.). In humans, various studies correlated DCS measures of cerebral blood flow with other physiological parameters in order to study various pathologies. CBF was correlated with invasive measures of brain tissue oxygen tension and cerebral microdialysis to study the brain in critically brain injured adults (Baker et al.), and blood pressure in ischemic stroke patients to study the impairment of autoregulation in this patient group (Gregori-Pla et al.). Another study on stroke monitored hemodynamic changes in stroke patients being treated with mechanical thrombectomy (Forti et al.), where DCS was shown to be a potentially useful clinical tool to improve patient outcome after stroke. Other clinical studies used DCS to measure skeletal blood flow in response to massage therapy (Matsuda et al.). The wide variety of clinical DCS research presented is perhaps a token of the future potential and clinical importance of this emerging technique.

Several studies using fNIRS within the topics of brain computer interfacing $(\mathrm{BCl})$ and hyperscanning highlighted a plethora of relatively novel applications. In the field of $\mathrm{BCI}$, fNIRS signals were used to control prosthetic limbs (Naseer et al.), as well as a means of communication with locked-in patients (Birbaumer). Joy Hirsch showed that fNIRS hyperscanning enables rigorous investigation of the study of the neural mechanisms between several brains communicating with each other during social interactions. fNIRS was used to study the synchronicity of functional activation during various hyperscanning paradigms such as speed-dating ( $\mathrm{Li}$ et al.), discussing recent news and media sensations (Wesner et al.), and performing competitive and cooperative tasks (Mizuno et al.). The cortical areas showing synchronicity during the various paradigms differed, yet the emerging data suggests that the left prefrontal area is important during social interactions.

To conclude the meeting, an election for new board members, ratified by the membership, took place. The next fNIRS meeting will be hosted by Maria Angela Franceschini, in Boston USA in 2020. Dr. Franceschini was also ratified as president-elect and will assume presidency of SfNIRS at the 2020 meeting. We are certain that both the size and the diversity of the meeting will continue to grow. 